\title{
BodyRating: Uma proposta para anotação de silhueta corporal para avaliação de autopercepção
}

\author{
Anderson S. Fonseca ${ }^{1}$, Victor H. B. de Lemos ${ }^{1}$, Artur A. Silva ${ }^{2}$ \\ Geraldo Braz Júnior ${ }^{1}$, Anselmo C. Paiva ${ }^{2}$, Antônio A. M. da Silva ${ }^{3}$ \\ ${ }^{1}$ Programa de Educação Tutorial em Ciência da Computação (PETComp) \\ ${ }^{2}$ Núcleo de Computação Aplicada (NCA) \\ ${ }^{3}$ Coortes RPS - São Luís - Hospital Universitário (HU-UFMA) \\ Universidade Federal do Maranhão (UFMA) \\ andsfelive.com, victorhbl12, astobiro@gmail.com@gmail.com,geraldo.braz, \\ anselmo.paiva, antonio.araujo@ufma.br
}

\begin{abstract}
Nowdays, the appearance is of great importance in the lives of many and the way people evaluate their own image can lead to several self-perceived disorders. Evaluate the level of these disorders led to the proposal of the BodyRating tool that notes the classification of $3 D$ models of voluntary patients made by specialists in the field taking into account the Scale of Silhouettes. The tool was presented and it was widely accepted. After the annotation of the $3 \mathrm{D}$ models, the collected data will be used as base to the development of an automatic classification tool that is a future proposal of help to specialists.
\end{abstract}

Resumo. Atualmente, a aparência é de grande importância na vida de muitos e a forma que pessoas avaliam a própria imagem pode levar a diversos transtornos de autopercepção. Avaliar o nível desses transtornos levou a proposta da ferramenta BodyRating que anota a classificação de modelos $3 D$ de pacientes voluntários feita por especialistas da área levando em consideração a Escala de Silhuetas. A ferramenta foi apresentada e percebeu-se uma grande aceitação da mesma. Após a anotação dos modelos 3D, os dados coletados serão utilizados como base para o desenvolvimento de uma ferramenta de classificação automática que é uma futura proposta de auxílio a especialistas.

\section{Introdução}

Diante desta época de informação, onde as pessoas em pouco tempo poderiam compartilhar o que pensam e o que fazem, as redes sociais se tornaram o palco para um grande acúmulo de opiniões. Assim, as pessoas começaram a se importar mais com a sua aparência, do que agrada ou não um público comum, modificando seu pensamento de como ela se mostra ao mundo. $\mathrm{O}$ fato do uso de redes sociais podem trazem uma série de transtornos sociais, como cybercrime, bullying, etc e vários transtornos pessoais, como anorexia, repressão social, depressão, etc.

A imagem corporal é a construção multidimensional que descreve amplamente as representações internas da estrutura corporal e da aparência física em relação a nós mesmos e aos outros [Damasceno et al. 2006] é um conceito de como um pessoa se percebe. Os problemas citados anteriormente podem afetar diretamente a autopercepção de um 


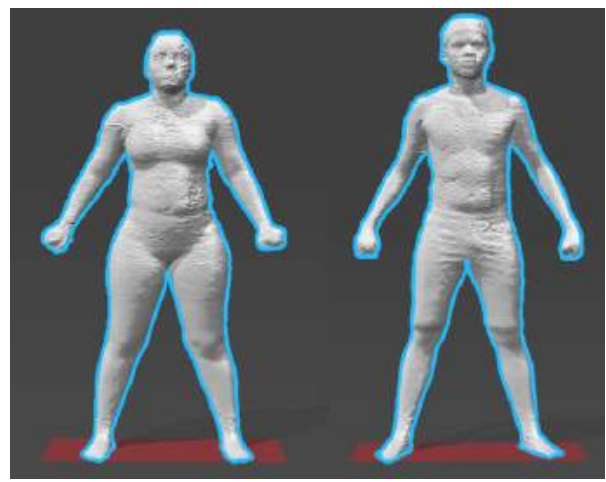

Figura 1. Exemplos de modelos 3D de voluntários do projeto Coortes RPS.

indivíduo. Dessa forma, é de grande importância uma forma de mensurar divergências entre a real imagem de uma pessoa e a sua auto imagem para melhor entendimento desses transtornos.

Com o intuito de verificar as modificações que a esta era de informação realiza na saúde das pessoas, foi criada o projeto Coortes RPS, consórcio de coortes brasileiras de nascimento de Ribeirão Preto, Pelotas e São Luís, com o intuito de pesquisar a saúde de jovens nascidos em 1997 e acompanhar a saúde dos mesmos até a chegada da vida adulta. Assim poderia se medir ou ter uma ideia de o quanto este impacto difere na formação de saúde do individuo.

Dentre os procedimentos feitos no Coortes RPS, a geração de modelos 3D dos pacientes (Figura 1) possibilita uma forma diferenciada de estudos de transtornos de autopercepção. Ao longo dos anos do projeto foram coletados mais de 4000 modelos e, levando em consideração as dimensões da base de dados, sentiu-se a necessidade de uma formar prática de classificar esses modelos quanto a real forma do corpo do paciente em questão. Assim, foi proposto a ferramenta BodyRating, uma aplicação web criada a fim de fazer anotação da classificação de cada paciente e gerar uma base de dados robusta para futuros estudos.

\section{Metodologia}

Partindo da ideia que o objetivo era desenvolver uma ferramenta simples e prática para anotação dos dados de cada indivíduo, era necessário uma métrica de classificação que proporcionasse tais condições. Dessa forma, foi escolhida a Escala de Silhuetas (Figura 2) [Stunkard et al. 2006] por já ser amplamente difundida no meio acadêmico e oferecer um grande número de classes em versões para ambos os gêneros.

A escala de silhuetas já foi utilizada em cegos congênitos para avaliar satisfação corporal onde foi criado uma versão bidimensional da mesma[da Rocha Morgado et al. 2009b].

A Figura 3 apresenta um esquema de funcionamento da metodologia proposta. Ela tem início com a autoavaliação de cada indivíduos seguido da captura de seus respectivos modelos 3D. Após dessas etapas preliminares, inicia-se a pré-classificação, uma sub-etapa da classificação, onde especialistas classificam a base de dados seguindo a escala de silhuetas. Em seguida ocorre a classificação computadorizada que usa os dados 


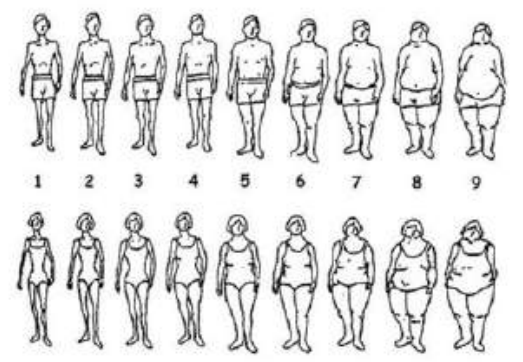

Figura 2. Escala de Silhuetas em suas versões masculina e feminina.

fornecidos pelos especialista para automatizar o processo. E por último, há a análise dos resultados obtidos.

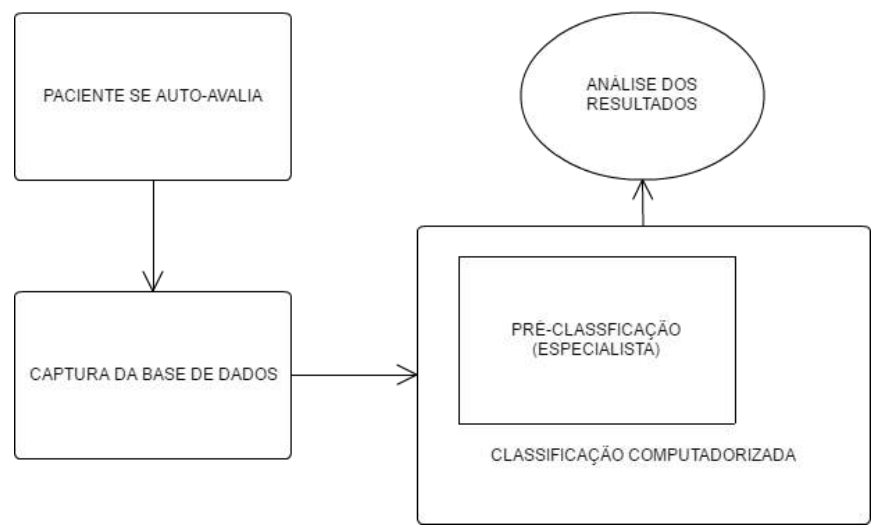

Figura 3. Fluxograma das etapas da metodologia aplicada

Usando a ferramenta BodyRating, no processo de classificação, são coletados a classe, gênero e um possível comentário que o especialista possa ter sobre o modelo. A aplicação da ferramenta acontece em duas etapas iniciais. Na primeira fase de testes, é realizada uma avaliação por 5 especialistas, sendo eles estudantes de medicina e fisiologistas, que avaliarão amostras de cerca de 300 modelos de paciente, com a finalidade de mostrar o quanto a perspectiva de especialistas se diferem a partir de um determinado modelo. Na segunda fase, os modelos avaliados serão comparados com as opiniões de seus respectivos pacientes, verificando o quanto a opinião dada por especialistas pode diferir da opinião própria do paciente.

\section{Resultados}

Foi apresentada a uma bancada de especialistas do projeto Coorte RTS a importância da avaliação de silhuetas e os possíveis transtornos que podem ser mostrados durante a avaliação dada por um médico especializado e um paciente. Foi então mostrada a aplicação BodyRating que tem o intuito de recuperar os dados das avaliações feitas por especialistas e processar esses dados para uma detecção de opiniões que divergem entre os mesmos, mostrando de maneira gráfica as opiniões que um médico tem sobre a silhueta do indivíduo e do paciente sobre sua silhueta, ambos baseadas na Escala de Silhuetas.

Com a aprovação da bancada, cinco voluntários foram selecionados para avaliar um quantidade específica de modelos, os resultados da avaliação são registrados e guarda- 
dos para realização de uma análise mais especifica, onde já é possível identificar os detalhes sobre os modelos disponibilizados pelo BodyScan e diferenciá-los de cada avaliação registrada.

\section{Conclusão e Direções Futuras}

Em suma, a problemática dos transtornos de autopercepção se mostrou de grande importância bem como o melhor entendimento da mesma. Além disso, ficou evidente a aceitação da ferramenta entre os especialistas consultados assim como a sua utilidade. Pode-se perceber também que não houve qualquer tipo de resistência à ideia de desenvolvimento de um classificador automático.

Como trabalhos futuros os modelos que irão ser avaliados, passarão por uma outro processo mais específico, usando as técnicas de reconhecimento padrões e análise de imagem, a aplicação BodyRating irá aprender sobre as avaliações de cada especialista, sendo assim a aplicativo em questão poderá automaticamente sugerir a partir do modelo 3D gerado qual a classificação na Escala de Silhuetas o paciente se enquadra, a sugestão não irá substituir a opinião do especialista, mas espera-se que a informação gerada pelo BodyRating sirva futuramente como uma segunda opinião para futuras avaliações.

\section{Referências}

Antunes, A. V., Pozzobon, A., and Pereira, A. L. B. (2011). Avaliação antropométrica, autopercepção corporal e perfil nutricional de mulheres adultas. Revista Destaques Acadêmicos, 3(3).

da Rocha Morgado, F. F., Ferreira, M. E. C., Andrade, M. R. M., and Segheto, K. J. (2009a). Análise dos instrumentos de avaliação da imagem corporal. Fitness \& performance journal, (3):204-211.

da Rocha Morgado, F. F., Ferreira, M. E. C., de Lima Alexandrino, D. F., and Amaral, A. C. S. (2009b). Escala de silhuetas bidimensionais para avaliação da satisfação corporal do cego.

Damasceno, V. O., Vianna, V. R., Vianna, J. M., Lacio, M., Lima, J. R. P., and Novaes, J. S. (2006). Imagem corporal e corpo ideal. Rev Bras Ciên e Mov, 14(1):87-96.

de Assis, C. L., Borine, B., Lacerda, S. R., and da Costa, A. (2013). Autoavaliação de peso corporal e classificação do índice de massa corporal de estudantes do ensino superior de cacoal-ro-doi: http://dx. doi. org/10.15603/2176-1019/mud. v21n2p30-39. Mudanças-Psicologia da Saúde, 21(2):30-39.

Stunkard, A., Sorensen, T., and Schlusinger, F. (2006). Use of danish adoption register for the study of obesity and thinness. 1983. Scagliusi FB et al.

Zenith, A. R., Marques, C. R. C., Dias, J. C., and Rodrigues, R. C. L. C. (2012). Avaliação da percepção e satisfação da imagem corporal em usuários do programa academia da cidade em belo horizonte minas gerais. e-Scientia, 5(1):09-17. 